\title{
Magnitude of Drug-resistant Enterococcus species from Intestinal Tracts of Hospitalized Pediatric Patients in J imma University Specialized Hospital, Southwest, Ethiopia
}

\author{
Gebrish S ${ }^{1}$, Fikadu $B^{1}$ and Asfaw $T^{2 *}$ \\ ${ }^{1}$ Department of Medical Laboratory Science, Debre \\ Berhan Health Science College, Debre Berhan, Ethiopia \\ ${ }^{2}$ Department of Medical Laboratory Science, College \\ of Medicine, Debre Berhan University, Debre Berhan, \\ Ethiopia \\ *Corresponding author: Tsegahun Asfaw, \\ Department of Medical Laboratory Science, College \\ of Medicine, Debre Berhan University, Debre Berhan, \\ Ethiopia
}

Received: December 26, 2018; Accepted: J anuary 18, 2019; Published: J anuary 25, 2019

\begin{abstract}
Background: Multi-drug-resistant enterococci are the major source of infection as well as nosocomial spread. There is scarcity of data on drugresistant enterococci in developing country including Ethiopia. Therefore, the present study aimed to determine the magnitude of drug resistant Enterococcus species from intestinal tracts of hospitalized pediatric patients at Jimma University specialized hospital.

Method: The study was conducted among hospitalized pediatric patients at Jimma University Specialized Hospital, from February 15 to March 25, 2016. Rectal swabs was collected and processed for bacterial isolation and susceptibility testing. The isolates was identified to species level by cultural characteristics, Gram's stain, catalase test and other biochemical tests. Susceptibility testing to antimicrobial agents was done using Kirby-Bauer disk diffusion method.
\end{abstract}

Result: Enterococci were isolated from 12 (23\%) of the study participants. The isolates were Enterococcus faecium (50\%), Enterococcus faecalis (33.3\%) and Enterococcus gallinarum (16.7\%). Among 12 tested Enterococci isolates, $5(41.7 \%)$ were resistant to ampicillin, $7(58.3 \%)$ to streptomycin, $6(50 \%)$ to gentamycin. $7(58.3 \%)$ to ciprofloxacin, $5(41.7 \%)$ to norfloxacin and $8(66.7 \%)$ to erythromycin. Multiple drug resistance was observed among $75 \%$ of $E$. faecium and $E$. faecalis. Vancomycin resistant Enterococci were observed in $16.7 \%$ of $E$. faecium isolates.

Conclusion: This study reveals high rate of fecal colonization by multidrugresistant enterococci and prevalence of vancomycin resistance strains. Thus periodic surveillance of antibiotic susceptibilities is recommended to detect emerging resistance and to prevent its spread.

Keywords: Magnitude; Drug-resistance; Enterococcus species; Pediatric Patients

\section{Introduction}

Enterococci are normal inhabitant of the gastrointestinal tract. However, they can also be significant pathogens causing several infections. The most common nosocomial infections caused by these organism are urinary tract infections (associated with instrumentations and antimicrobials administration) [1]. The emergence of vancomycin resistance enterococci (VRE) is a global issue due to few option left for disease management. Besides drug resistant Enterococci can colonize the intestinal tract of hospitalized patients and become major source of infection as well as nosocomial spread $[2,3]$.

In humans, enterococcal infections may be caused by at least 12 species but most clinical infections are due to either Enterococcus faecalis or E. faecium. E. faecalis is the most common cause (80-90 \%) followed by E. faecium (10-15 \%). Occasional infections are due to Enterococcus gallinarum, Enterococcus raffinosus, Enterococcus casseliflavus, Enterococcus avium, Enterococcus pseudoavium, Enterococcus malodoratus, Enterococcus mundtii, Enterococcus durans, and Enterococcus hirae [4]. The proportion of isolates of motile Enterococci (E. gallinarum, E. casseliflavus) is low. But they are intrinsically resistant to vancomycin and inappropriate treatment may contribute to morbidity and mortality [5].

Several studies have documented that enterococcal infections are most commonly caused by the patient's own commensal flora. Colonization may occur long before or immediately before infection, but it plays a major role in the development of nosocomial infection [2]. Despite the importance of these etiologic agents there is dearth of information regarding antimicrobial resistance of Enterococcus species isolated from intestinal tract of hospitalized patients in Ethiopia. Thus, the present study will be conducted to determine antimicrobial resistance pattern of fecal enterococci isolates from 
hospitalized pediatric patients.

\section{Methods}

\section{Study area}

The study was conducted at Jimma university specialized hospital, Jimma town, south west Ethiopia. It is located $352 \mathrm{~km}$ southwest of Addis Ababa. It provides services for approximately 9000 inpatients and 80000 outpatients each year with bed capacity of 450 beds. According to the national 2015 census, this town had a total population of 120,960 of whom 60,824 were men and 60136 were women.

\section{Study design and periods}

Cross sectional study was conducted to determine the magnitude of drug resistance Enterococcus species from intestinal tracts of hospitalized Pediatric patients aged 0 to 15 years from February 15 to march 25, 2016

\section{Sampling technique and sample size}

52 patients, who had at least 2 days of hospital stay at Pediatric ward of Jimma University specialized hospital were enrolled by using convenience sampling technique.

\section{Inclusion and exclusion criteria}

Pediatric patients aged 0 to 15 years and having at least 48 hour hospital stay during the study period and fulfill the inclusion criteria was included while who cannot respond to the interview and for children without getting permission from guardian and consent was excluded.

\section{Data collection techniques}

Scio-demographic data was collected by pretested questionnaires after obtaining a written informed consent from study participants. Fecal samples were collected in sterile plastic stool containers and then were transferred to the laboratory. From critically ill patients rectal swabs were collected using sterile cotton swab moistened in sterile normal saline solution at intensive care units. Then, the swabs were immersed in well-labeled Cary-Blair semi-solid medium prepared in screw-capped tubes.

Culture and identification: Stool specimens and rectal swabs were inoculated onto Enterococci selective media [Bile Esculin azide agar plates with and without $6 \mu \mathrm{g} / \mathrm{ml}$ of vancomycin to recover vancomycin-susceptible isolates and incubated at $37^{\circ} \mathrm{C}$ for $24 \mathrm{~h}$. Colonies showing macroscopically morphological differences and whose colony morphology was consistent with that of Enterococci [colonies with colourless or grey and surrounded by a black halo (hydrolysis of esculin)] were subcultured and identified as Enterococci by additional tests (gram stain, catalase test, $6.5 \% \mathrm{NaCl}$ test, growth at $45^{\circ} \mathrm{C}$ and motility test) as recommended by Facklam and Collins [6], Manero and Blanch [7]. Identification of these isolates to species level was performed by API-20 Streptococcus system (bioMe'rieux). For further identification, stock cultures were stored at BHI Broth containing $50 \%$ glycerol at $-20^{\circ} \mathrm{C}$.

Antibiotic susceptibility testing: Antimicrobial susceptibility studies were performed by disc diffusion (Kirby-Bauer) method according to Clinical Laboratory Standards Institute (CLSI) for different tested drugs.

\begin{tabular}{|c|c|c|c|c|}
\hline Variable & $\begin{array}{c}\text { Culture Positive } \\
\text { n (\%) }\end{array}$ & $\begin{array}{c}\text { Culture Negative } \\
\text { n (\%) }\end{array}$ & Total (\%) & P-value \\
\hline \multicolumn{4}{|c|}{ Age category in year } & \\
\hline 0-7 year & $8(25.8 \%)$ & $23(74.2 \%)$ & $31(59.6 \%)$ & \multirow{2}{*}{0.432} \\
\hline 8-15 year & $4(19 \%)$ & $17(81 \%)$ & $21(40.4 \%)$ & \\
\hline \multicolumn{4}{|l|}{ Sex } & \\
\hline Male & $7(26 \%)$ & $20(74 \%)$ & $27(52 \%)$ & \multirow{2}{*}{0.546} \\
\hline Female & $5(20 \%)$ & $20(80 \%)$ & $25(48 \%)$ & \\
\hline \multicolumn{4}{|c|}{ Hospital duration } & \\
\hline 2-15 day & $3(10.7 \%)$ & $25(89.3 \%)$ & $28(53.8 \%)$ & \multirow{2}{*}{0.135} \\
\hline$>15$ day & $9(37.5 \%)$ & $15(62.5 \%)$ & $24(46.2 \%)$ & \\
\hline \multicolumn{4}{|c|}{ Previous antibiotic treatment } & \\
\hline Yes & $7(17.1 \%)$ & $34(82.9 \%)$ & $41(78.8 \%)$ & \multirow{2}{*}{0.313} \\
\hline No & $5(45.5 \%)$ & $6(54.5 \%)$ & $11(21.2 \%)$ & \\
\hline
\end{tabular}

\section{Data processing, analysis}

The collected data was clearly summarized, filled and processed by using SPSS version 21. The data was analyzed by using SPSS version 21. Descriptive statistics was employed to examine the finding, and the result was presented by using tables, charts and graphs. The association between independent and dependent variables was determined. P-value less than 0.005 was considered as statistically significant value.

\section{Quality assurance}

The questionnaires was pretested a week before actual data collection. The quality of reagents and equipment was checked and used according to manufacturer directions. The data was collected by trained data collectors and the result was recorded carefully and correctly. Standard operating procedure was applied during specimen collection, culture, drug susceptibility test and biochemical test.

\section{Ethical consideration}

The study protocol was reviewed and approved by the ethical and review committee of Jimma University. For all study participant the objective of the study was explained and written informed consent was obtained. Those positive for enterococcus was referred to their respective clinicians for further management.

\section{Results}

\section{Demographic characteristics}

Among 52 participants, 27 (52\%) were males and 25 (48\%) were females. The mean age of the patient's was 3 years. $78.8 \%$ study participant had a history of exposure to one or more antimicrobial agent in the last 2 weeks and $21.2 \%$ were without exposure and the average hospital stay was 17.5 days with a range of 2-45days (Table 1).

\section{Enterococci isolates}

Among all participants, 12 (23\%) of the study participants were positive for at least one Enterococcus spp. There was no statistically significant association between isolation of Enterococci with age, sex, hospital duration and antibiotic history (Table 1).

\section{Species distribution}

The distribution of species is that a total of 12 enterococcal 
Table 2: Antibiotic resistance profile of Enterococcus species by Kirby-Bauer disc diffusion method from intestinal tract of Hospitalized pediatric patients in JUSH, Ethiopia.

\begin{tabular}{|c|c|c|c|c|}
\hline \multirow{2}{*}{ Antibiotics } & \multicolumn{4}{|c|}{ Resistant isolates (\%) } \\
\cline { 2 - 5 } & $\begin{array}{c}\text { E. faecalis } \\
(\mathbf{n}, \mathbf{4})\end{array}$ & $\begin{array}{c}\text { E. faecium } \\
(\mathbf{n}, \mathbf{6})\end{array}$ & $\begin{array}{c}\text { Other species } \\
(\mathbf{n}, \mathbf{2})\end{array}$ & Total (n,12) \\
\hline Ampicillin & $\mathbf{1 ( 2 5 \% )}$ & $4(66.7 \%)$ & $0(0 \%)$ & $5(41.7 \%)$ \\
\hline Penicillin & $4(100 \%)$ & $5(83.3 \%)$ & $1(50 \%)$ & $10(83.3 \%)$ \\
\hline Gentamicin & $2(50 \%)$ & $4(66.7 \%)$ & $0(0 \%)$ & $6(50 \%)$ \\
\hline Ciprofloxacin & $3(75 \%)$ & $4(66.7 \%)$ & $0(0 \%)$ & $7(58.3 \%)$ \\
\hline Streptomycin & $2(50 \%)$ & $4(66.7 \%)$ & $1(50 \%)$ & $7(58.3 \%)$ \\
\hline Erythromycin & $1(25 \%)$ & $5(83.3 \%)$ & $2(100 \%)$ & $8(66.7 \%)$ \\
\hline Norfloxacin & $1(25 \%)$ & $3(50 \%)$ & $1(50 \%)$ & $5(41.7 \%)$ \\
\hline Vancomycin & $0(0 \%)$ & $1(16.7 \%)$ & $0(0 \%)$ & $1(8.3 \%)$ \\
\hline
\end{tabular}

isolates were obtained from 52 patients. The commonly enterococcal isolates were E. faecium (50\%) followed by E. faecalis (33.3\%) and $E$. gallinarum (16.7\%).

\section{Antimicrobial resistance of Enterococcal isolates}

B Lactam resistance: $1 / 4(25 \%)$ E. faecalis and $4 / 6(66.7 \%)$ E. faecium were resistant to ampicillin. All E. fecalis and 5/6 (83.3) E. faecium isolates were resistant to penicillin. 1/2 (50\%) occasional Enterococcus species (E. gallinarum) were resistant to penicillin.

Aminoglycoside resistance: High-level resistance to gentamicin and streptomycin was detected by the high content disk. Gentamycin resistant were observed in $50 \%$ of E. faecalis and $66.7 \%$ of $E$. faecium. $2 / 4$ (50\%) E. faecalis and $66.7 \%$ E. faecium were resistant to streptomycin.

Vancomycin-resistant Enterococci: E. faecalis isolates was not resistance to vancomycin while 1 (16.7\%) of E. faecium isolates were resistant to vancomycin (Table 2).

\section{Discussion}

The rapid emergence of resistance in enterococci and the increasing incidence of colonization and infection with VRE have become health care issues. This study investigated the prevalence of Enterococci and antibacterial resistance patterns of enterococci isolated from fecal samples of hospitalized pediatrics patients. In this study the distribution of enterococcus isolates were E. faecium (50\%), E. faecalis (33.3\%) and E. gallinarum (16.7\%). This is comparable to the study done in Brazil. But disagreement with reports from United States where E. faecalis was predominant over E. faecium [8]. In this study, the predominant enterococcus isolates was E. faecium. Study in Singapore has also reported an increase in E. faecium from 78.9 to $91.8 \%$ over a period of 5 years from 2006 to 2010 from clinical cultures [9]. Another study from India has also reported $66 \% E$. faecium from blood sample [10]. In this study the prevalence of $E$. gallinarum was $16.7 \%$ which is higher than study done in Ethiopia [1]. This might be due to, though Enterococci including E. gallinarums are infrequently isolated from clinical specimens, they have been implicated in a wide variety of invasive infections in humans, especially immunocompromised or chronically ill patients.

In this study, E. faecalis isolates showed $25 \%$ resistance rate to ampicillin. It is higher than the resistance rates reported in Kuwait,
Hong Kong and Brazil, which is 0-8.3 \% [11-13], and lower than 60.7\% reported from Gaza [14]. Resistance rates to ampicillin was observed in $66.7 \%$ of E. faecium isolates which is comparable with study done in Gaza 66.7\% [14]. However, lower than study reported from Israel (87.5\% resistance rate) [15]. All E. facalis and $83.3 \%$ E. faecium isolates were resistant to penicillin which is similar to study done in India from clinical isolates [10]. The reason for higher prevalence of $\beta$-lactam antibiotic resistance in this study might be due to chronic cases and wider usage of broad spectrum antibiotics relative or enterococcus isolates possess an intrinsically relative resistance to penicillin and ampicillin. Furthermore, E. faecium is less susceptible to $\beta$-lactam agents than $E$. faecalis because their penicillin-binding proteins (PBPs) have lower affinities for these antibiotics and some strains have plasmid-encoded $\beta$-lactamase.

Aminoglycosides are frequently used in combination with cell wall active antibiotics for severe enterococcal infections. Since enterococcal resistance to gentamicin and streptomycin occurs by different mechanisms, it is important to test susceptibility to both agents. Enterococci with high level resistance to streptomycin are susceptible to gentamicin. And also gentamicin resistance is a good predictor of resistance to other aminoglycosides except streptomycin [16]. In this study, E. faecalis and E. faecium showed resistance for many drugs. Concomitant resistance of high level aminoglycoside resistance (HLAR) strains to the $\beta$-lactam antibiotic (ampicillin) was quite higher (25\% of E. faecalis and $66.7 \%$ of E. faecium strains). This finding is a cause of concern, because the synergistic activity of the combination of $\beta$-lactam antibiotics with HLAR in the treatment of enterococcal infections is totally abolished. In such instances, controlling the spread of these organisms have supreme importance.

In this study $58.3 \%$ of enterococci isolate were resistant to ciprofloxacin and $41.7 \%$ of the isolates were resistant to norfloxacin. Other alternative antibiotics to treat infection by enterococcus also showed high rates of resistance (erythromycin (66.7\% of resistance) and $(58.3 \%)$ of resistance to streptomycin). The high rates of resistance in present study might be due to excessive or inappropriate use of those antibiotics for empirical treatment of mixed nosocomial infections caused by enterococci.

The emergence of VRE is also due to the inappropriate use of cephalosporin as well as poor hospital infection control measures. This study showed $16.7 \%$ E. faecium resistant to vancomycin which is higher than study done in Egypt 4\% [17], Iran 6.2\% [18], South Africa $10.2 \%$ [19] and report from Korea 12\% [20] and lower than report from Turkey $34.8 \%$ [21]. The possible reason for the emergence of VRE in this study might be due to antibiotic selective pressure because the patients had long duration in hospital and high rate of antibiotics treatment.

The increase of invasive infections caused by multi resistant $E$. faecium, however, did not only increase the total burden of nosocomial enterococcal infections, but also resulted in a partial replacement of E. faecalis by E. faecium as a cause of hospital-associated infections. Several studies showed that an increased proportion of nosocomial enterococcal infections caused by E. faecium.

\section{Conclusions and Recommendations}

$23 \%$ of the hospitalized pediatrics patients carried enterococci 
in their gastrointestinal tracts and E. faecium was the predominant species. E. faecium showed highest resistance rate to vancomycin. Therefore, regular monitoring for the presence of VRE in both hospitals and the community, effective strategies for the prevention of antimicrobial resistance should be practiced. Unnecessary use of antibiotics and ignorance infection control measure should be stopped.

\section{Funding}

This work was funded from Jimma University core budget.

\section{Authors' Contributions}

SG-performed the laboratory activities. BF, SG and TA- analyzed the data. TA-wrote the manuscript. All authors read and approved the final manuscript.

\section{Acknowledgment}

We would like to thank Jimma University for giving this opportunity and availing the resources. We also thank staffs and head of Jimma university specialized hospital for their help during data collection process.

\section{References}

1. Abamecha $\mathrm{AH}$, Wondafrash $\mathrm{B}$ and Abdissa A. Antimicrobial resistance profile of Enterococcus species isolated from intestinal tracts of hospitalized patients in Jimma, Ethiopia. BMC Res Notes. 2015; 8: 213.

2. Boost M, Lai L, O'Donoghue M. Drug resistance in fecal enterococci in Hong Kong. J Infect Chemother. 2004; 10: 326-330.

3. Arias CA, Murray BE. Emergence and management of drug-resistant Enterococcal infections. Expert Rev Anti Infect Ther. 2008; 6: 637-655.

4. Sood S, Das MM, Kapil A. Enterococcal infections and antimicrobial resistance. Indian J Med Res. 2008; 128: 111-121.

5. Shah L, Mulla S, Patel KG, Rewadiwala S. Prevalence of enterococci with higher resistance level in a tertiary care hospital: A matter of concern. Natl J Med Res. 2012; 2: 25-27.

6. Manero A, Blanch AR. Identification of Enterococcus species with a biochemical key. Appl Environ Microbiol. 1999; 65: 4425-4430.

7. Clinical and Laboratory Standards Institute. Performance standards fo antimicrobial susceptibility testing, informational supplement (M100-S17), $17^{\text {th }}$ edn. Clinical and Laboratory Standards, Wayne. 2007.
8. Silverman J, Thal LA Perri MB, Bostic G, Zervos MJ. Epidemiologic evaluation of antimicrobial resistance in community-acquired Enterococci. J Clin Microbiol. 1998; 36: 830-882.

9. Cai $Y$, Chan PJ, Fisher D, Hsu L, Koh T, Krishnan P. Vancomycin-resistan Enterococci in Singaporean hospitals: 5-year results of a multi-centre surveillance programme. Ann Acad Med Singapore. 2012; 41: 77-81.

10. Kapoor L, Randhawa VS, Deb M. Antimicrobial resistance of enterococcal blood isolates at a pediatric care hospital in India. Jpn J Infect Dis. 2005; 58 : 101-103.

11. Udo E, Al-Sweih N, John P, Chugh TD. Antibiotic resistance of enterococci isolated at a teaching hospital in Kuwait. Diag Microbiol Infect Disn. 2002; 43: $233-238$

12. Boost M, Lai L, O'Donoghue M. Drug resistance in fecal enterococci in Hong Kong. J Infect Chemother. 2004; 10: 326-330.

13. Eduardo E, Freitas A, Reiter K, Luts L, Afonso Barth A. Identification, antimicrobial reistance and genotype characterization of Enterococcus spp. in Porto Alegre, Brazil. Braz J Microbiol. 2009; 40: 693-700.

14. Elmanama AA, Al-Hindi A. Vancomycin-Resistant Enterococci in Feca Samples from Hospitalized Patients and Non-Hospitalized Individuals in Gaza City. 2009; 17: 243-249.

15. Dan M, Poch F, Leibson L, Smetana S, Priel I. Rectal colonization with vancomycin-resistant enterococci among high-risk patients in an Israeli hospital. J Hosp Infect. 1999; 43: 231-238.

16. Sood S, Das MM, Kapil A. Enterococcal infections and antimicrobia resistance. Indian J Med Res. 2008; 128: 111-121.

17. Kholy AE, Baseem H, Hall GS, Procop GW, Longworth DL. Antimicrobia resistance in Cairo, Egypt 1999-2000: A survey of five hospitals. J Antimicrob Chemother. 2003; 51: 625-630.

18. Assadian O, Askarian $M$, Stadler $M$, Shaghaghian S. Prevalence of vancomycin-resistant enterococci colonization and its risk factors in chronic hemodialysis patients in Shiraz, Iran. BMC Infect Dis. 2007; 7: 52

19. Von Gottberg A, van Nierop W, Duse A, Kassel M, McCarthy K, Brink A Epidemiology of glycopeptide-resistant enterococci colonizing high-risk patients in hospitals in Johannesburg, Republic of South Africa. J Clin Microbiol. 2000; 38: 905-909.

20. Yang J, Lee D, Kim Y, Kang B, Kim K, Ha N. Occurrence of the van genes in Enterococcus faecalis and Enterococcus faecium from clinical isolates in Korea. Arch Pharm Res. 2007; 30: 329-336.

21. Coleri A, Cokmus C, Ozcan B, Akcelik M, Tukel C. Determination of antibiotic resistance and resistance plasmids of clinical Enterococcus species. J Gen Appl Microbiol. 2004; 50: 213-219.
J Bacteriol Mycol - Volume 6 Issue 1 - 2019

ISSN : 2471-0172 | www.austinpublishinggroup.com

Asfaw et al. (C) All rights are reserved
Citation: Gebrish S, Fikadu B and Asfaw T. Magnitude of Drug-resistant Enterococcus species from Intestinal Tracts of Hospitalized Pediatric Patients in Jimma University Specialized Hospital, Southwest, Ethiopia. J Bacteriol Mycol. 2019; 6(1): 1094 